\title{
Study and Application of Electronic Records in the Pharmaceutical Equipment
}

\author{
Ning SHI ${ }^{1, a}$, Hui LIU ${ }^{2, b}$ and Yujuan LIU ${ }^{3, c}$ \\ ${ }^{1}$ Beijing Polytechnic, china \\ ${ }^{2}$ AVIC Beijing Aeronautical Manufacturing Technology Research Institute, china \\ ${ }^{3}$ Beijing Polytechnic, china \\ asn626sky@sohu.com, bwapman@tom.com, ' $13366603007 @ 163 . c o m$,
}

Keywords: GMP; Wet Granulation; Electronic records; Audit software

\begin{abstract}
The drug quality safety is a issue that governments have attached great importance to. The Chinese version of the CMP puts forward strict requirements of " traceability " in the pharmaceutical production process for the pharmaceutical industry.This paper mainly introduces how to realize the electronic record problems and solving method in the production of pharmaceuticals and for automatic control system of wet granulating machine, for meet the "traceability" requirement of the new version of GMP.The electronic record meets the requirements of GMP in pharmaceutical process,so as to ensure the quality of medicines, and achieved good production effect in practical production.
\end{abstract}

\section{Introduction}

In recent years, the food and drug safety has been paid more attention to, especially of the drug quality safety is related to people's life.Quality and safety risks of drug mainly have design quality risk (research, clinical trials), production quality risk (raw materials, production and storage) and the drug quality risk (misuse in use). Human factor had great influence on the quality risks in the production process. For a highly automated enterprise,it should be as much as possible to reduce the human factor to the minimum, so as to ensure the quality safety of drugs in the production process[1].Chinese new version of the GMP----- " Good Manufacturing Practice for Pharmaceutical Products " (Amended in 2010) has made detailed provisions in the drug quality safety of the pharmaceutical industry. The operation in the production process of drugs must be traceable, the same as that the automation system must conform to the requirements of electronic signature and electronic records, and raise a detailed claim on the contents of electronic record[2].

Therefore, as pharmaceutical equipment------the electrical design of granulating machine must also meet the requirements of the GMP on the quality safety production of drugs. As a part of quality control of drugs, if pharmaceutical equipment don't meet the GMP requirements for the quality control, it will be the elimination of pharmaceutical equipment industry. This paper introduces the implementation method of electronic record and electronic signature ,how to implement GMP for "traceability" request in the granulator.

\section{The Requirements of New Version GMP on Electronic Records for Pharmaceutical Enterprises}

Since implementation of the Chinese new version of GMP in 2011 March, all pharmaceutical companies are upgrading their equipment to meet requirements of the new version GMP for the pharmaceutical equipment. GMP put forward three requirements of pharmaceutical equipment automation system: continuous control, online monitoring, ease of use and traceability, the online monitoring and traceability are related to the electronic record and electronic signature[3].

In the new version of the GMP specification has respectively made detailed provisions on the personal access of electronic records, operation traces, recorded content and the duration of the record in the 162nd,163,171,172 of eighth chapters. The purpose of these requirements is to be 
"traceable" in the pharmaceutical production process, each operation can be clearly recorded in the system.The pharmaceutical companies can effectively find the reason of the drug quality safety problem, to aviod contamination and cross contamination in the process of pharmaceutical production,and to prevent confusion and human error, to ensure continuous and stable production the drug[2].

\section{The Connotation and Importance of Electronic Records}

In the pharmaceutical equipment, the idea mode of control should be accord with GMP management requirements, with the combination of automation and information technology, as far as possible to constraints, and replace the human actions, to realize the automation of control and management of production process. Quality should be controlled and can be traced in each stage of drug R \& D,production and circulation ,especially in the stage of production.To reduce the human intervention and record the production process, it is meaningful for the management of quality of medicines, and is an important basis for the analysis and continuous improvement of quality system. The records management is an important part of the GMP file management.

"Electronic record, electronic signature (ER/ES) " the relevant provisions of regulations come into effect, which U.S. Food and Drug Administration (FDA) issued the 21CFR eleventh part in August 20, 1997. This regulation of the electronic signature and electronic records is enforced.Purpose of this regulation is to provide the convenience for the product process of drugs introduction of electronic technology,to provide useful and practical guidelines.It elaborated how to replace the written form by electronic form[4].

In the new version of the GMP defined electronic records in detail, it include a mixture of any text, charts, data, voice, graphic or other performance information in electronic form, its establishment, modification, maintenance, archiving, retrieval or distribution is accomplished by computer system. Another concept associated with it is the electronic signature,it refers to arbitrary symbols or a series of symbolic compilation using computer data which performed by one person or approved as the personal handwritten signature with the same legal force.Strictly speaking, the electronic signature is the effective means for the management of electronic records, it should belong to the category of electronic record. [5].

There are three sources of electronic records in the production of pharmaceuticals[6]:

1. the operator manual input data, such as the name of the drug, batch number etc.

2. the system automatically generated record collection, such as temperature, humidity

3. the formation of archives by records processing.

According to the meaning of electronic records and the characteristics of the pharmaceutical equipment production process, granulator needs record data of five aspects: 1 . the operator manual input content, such as batch and product code;2. the parameter values of the running equipment, such as time, speed, current, and quantity of binder;3. Equipment operation stage, such as dry mixing,addition of binder,granulating;4. login information;5. operating record.

The new version of the GMP provide standard.According to this standard ,FDA considers the electronic record, electronic signature, the hand signature of the electronic records is trusted and reliable ,generally equivalent to paper records and handwritten signatures on paper.For the pharmaceutical industry, the characteristics of drug production decide that to ensure good product quality must by using process records. We can find out the causes of the sketchy product using the data of process record,this is essential to trace the drug quality problems. At the same time, along with the new version of GMP has issued and the application of computer system in the production of drugs, the use of electronic records is becoming more and more important[2].

\section{The Current Existing Problems in the Pharmaceutical Electronic Records}

With the improvement of people's living standards, the drug quality safety is in great price, but in recent years, there are some adverse drug reaction due to substandard medicines, so that people lost trust on the pharmaceutical companies. After all, the companies cannot be traced to the drug 
accident reasons after the accident,and cannot find principal for the drug accident. The reason is the operaor did not record the related information in pharmaceutical production on time, or record information is lost or deleted because of a hardware or human factors, even to fabricated false records for inspection, which seriously affect the quality problems in the production of pharmaceutical enterprises. Therefore, in order to improve the drug quality of pharmaceutical enterprises, the pharmaceutical enterprises should be strengthen the supervision in the pharmaceutical process, it is imperative for the pharmaceutical industry to introduce the electronic records in the automation system.

\section{Improved Methods of Electronic Records in the Granulator}

In order to improve the drug quality to meet the new GMP "traceability" requirement, according to the hardware characteristics of the granulator, I used two methods: the first is that use the classification management way to realize the electronic signature and electronic records in Proface touch screen and Mitsubishi PLC control system, as shown in Figure 1.Electronic records can record the login time, operation and operation time, and alarm records, the record can be printed and signed manully.At the same time, electronic and manual way coexistence. This is also consistent with the provisions of the GMP.

- The paper records and electronic records coexistence.

- The paper records and the electronic signature coexistence.

- Handwritten signature and electronic records coexistence[5].

In the drugs production enterprise in China, the first and second kind of situation is widespread.For example,the original data of many pharmaceutical enterprises is stored in electronic form,but records with GMP management significance is the printed paper medium with written signature.For this method of Proface screen and Mitsubishi PLC system we used the following way to realize it:

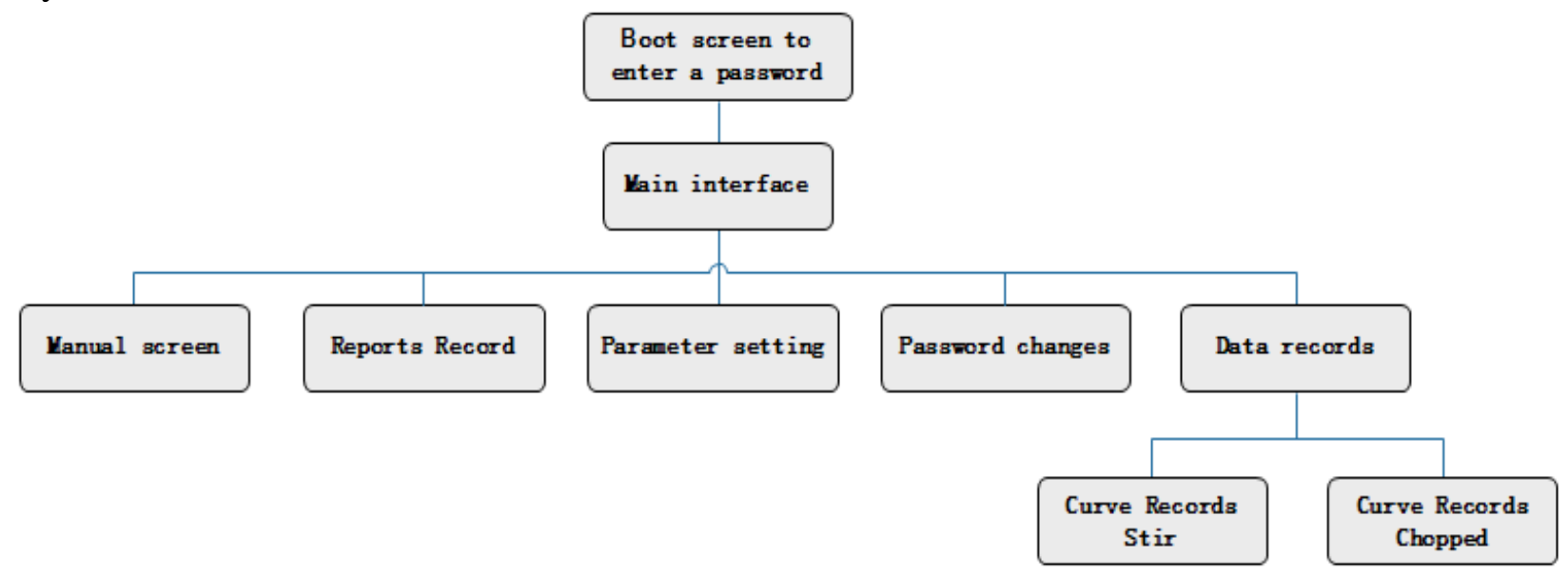

Fig1. The picture of the implementation of a new version GMP

This method just have restrictions on Staff who have privileges or not, when the operator access to the system, he can perform various operations on the system, it is dangerous for the drug product.In the new version of the GMP (2010 version), 163rd including the file management of the eighth chapter proposed that only authorized personnel can enter or change the data,and the action of changing and deleteing should be recorded in electronic data processing system.Operator should use a password or other means to control the system's login,and be reviewed independently by others after key data input[2]. Therefore the three-level management mode is adopted, the staff is divided into three types: technician, operator and manager. Technician which was awarded their-self code and password,can be allowed to enter the technician system. Technicians have permissions to set parameters, such as the process parameters for mass production, drug batch number and debugging test equipment and test new product parameters permissions,and the manual control permissions. In the process of operating the equipment, technicians code, equipment 
information and the date and time will be recorded.The operator which was awarded their code and password,can be allowed to enter the operator system. The operator can operate equipment to mass production just with the process parameters have been set by technicians.Similarly in the process of operating the equipment, operator code, equipment information and the date and time will be recorded too. The administrator which was awarded to manage the operator/technician's code and password, to maintain the uniqueness of each code and password combination, also ensure that no two people have the same code and password. Through the improvement with the new version of GMP requirements, the interface of the improved framework as shown below:

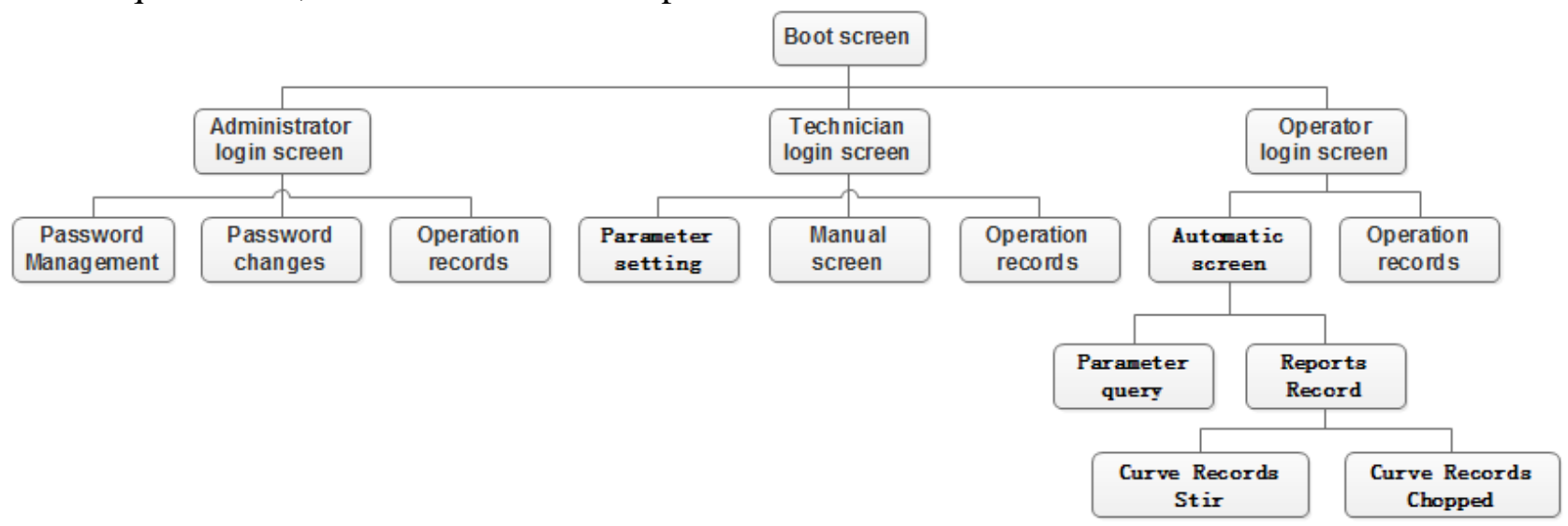

Fig2. Level 3 password management

The second method is to use the Siemens touch screen and PLC, at the same time with the Audit software to realize the electronic signature and electronic record. This model can be implemented personnel login, operations and the corresponding time to save in the form of electronic records, the record is save TXT document to facilitate the preservation of data, query and analysis. This way you can also ensure that if the data has been artificially altered,it can easily be queried by Audit to ensure the accuracy of the data.

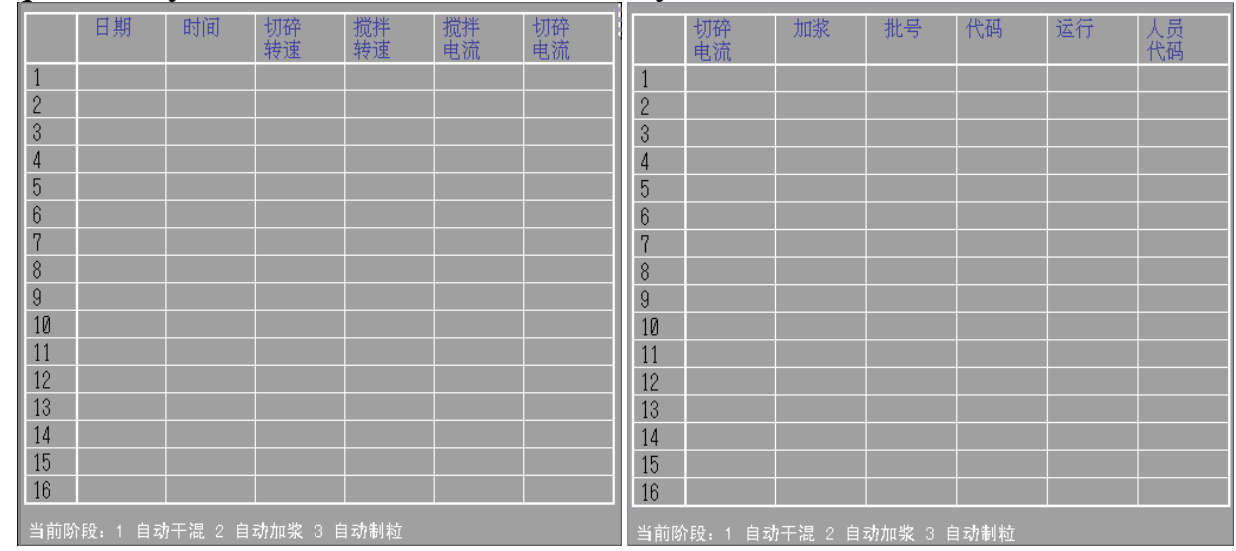

Fig3. The Equipment Operating Data

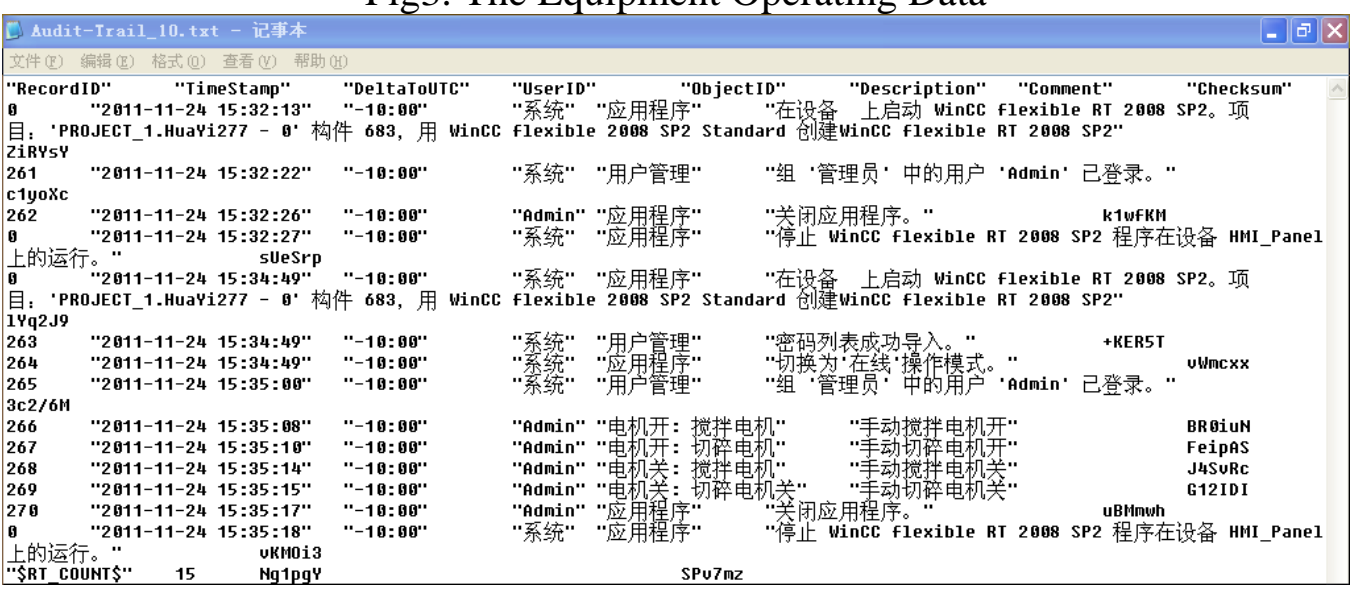

Fig4. the electronic records of Siemens system 
According to the electronic record, we can query the user login and logout time,and query the user's various operations of equipment .It provides a basis for the quality traceability .Meanwhile, the document can be queried, copy and delete,it is very easy to save and backup.

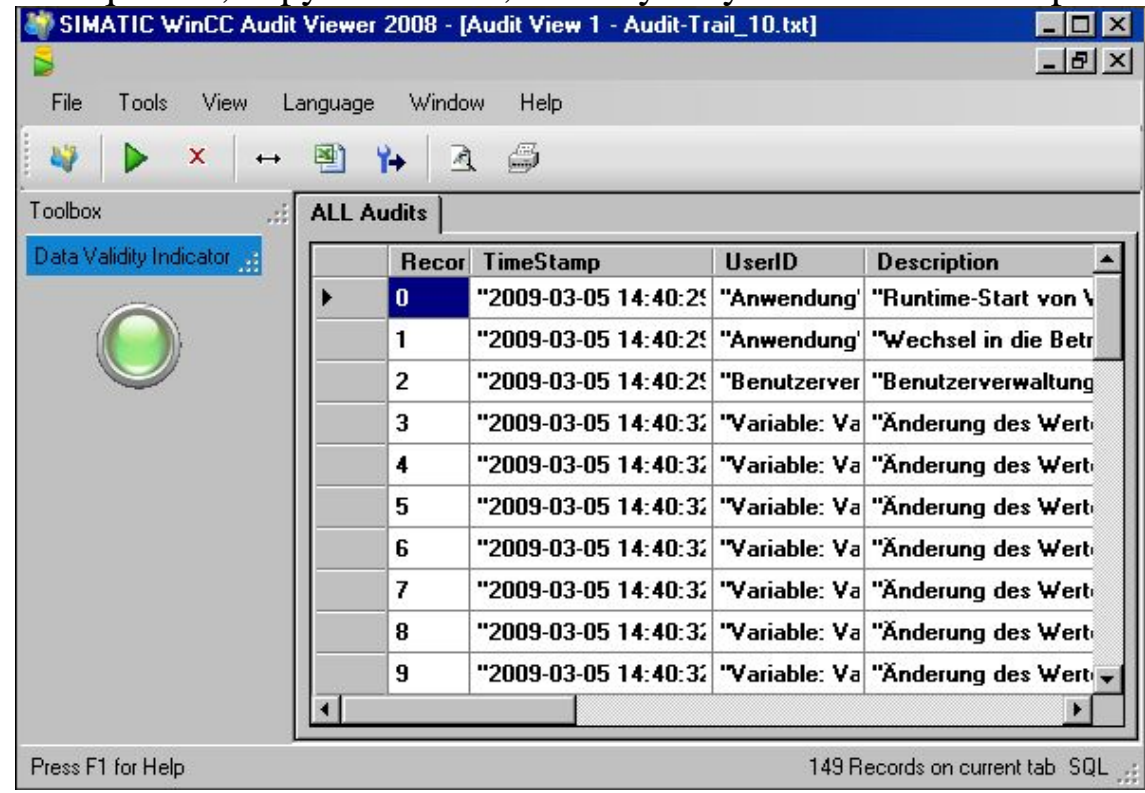

Fig5. Audit detection of document

\section{Conclusion}

The new version of GMP require each enterprise should have good production quipment,reasonable production process,and perfect quality management and strict inspection system, especially put forward detailed requirements on file management. In fact the purpose of these requirements is to ensure the reliability,controllbility and tracebility of drug quality factors in each stage of the entire life cycle of the drug, from $\mathrm{R} \& \mathrm{D}$, production, management, clinical, circulation and service .According to the actual hardware characteristics of granulating machine. Improved the original system, and proposed three level password and operation record combination,to solve data records prone to loss, fraud in the process of drug production ,and meet the GMP requirements for product traceability, improved the technical level of the granulator, so that it is at leading level in the domestic.

\section{References}

[1] Judith K Jones,Elyse E Kingery,Jing Tao and Jeff J Guo,Pre-marketing Drug Safety Risk Management in the Pharmaeeutieal Industry.Chin J Pharmacoepidemiol. Forum Vol.22-5(2013) ,p. 266-273.

[2] Good Manufacturing Practice for Pharmaceutical Products (Amended in 2010)SDA Order $\# 79,2011$

[3]Zhu Weigen. The Characteristics and Application of Electronic Data Processing System in Pharmaceutical Production[J].Mechanical and Electrical Information. Forum Vol. 29(2011),p.49-52

[4]Department of Health and Hervices.Food and Drug Administration.21 CFR Part11,Electronic Records;Electronic Signatures;Final Rule,March 20,1997

[5] Liu Fang and Feng Guozhong,Brief Discussion about the Main Problems for Electronic Records Management, Mechanical and Electrical Information.Forum Vol. 29 (2012),p.22-25

[6]Liang Yi,Brief Analysis of GMP Administration of Electronic Records.Pharmaceutical \& Engineering Design. Forum Vol. 29-3(2008),p. 25 28 


\section{Author:}

Shi Ning(1981.07), Female, Master ,Automation Engineering Institute of Beijing Polytechnic Research direction:The application of control theory in process control

TEL:15210337710

Email: sn626sky@sohu.com 